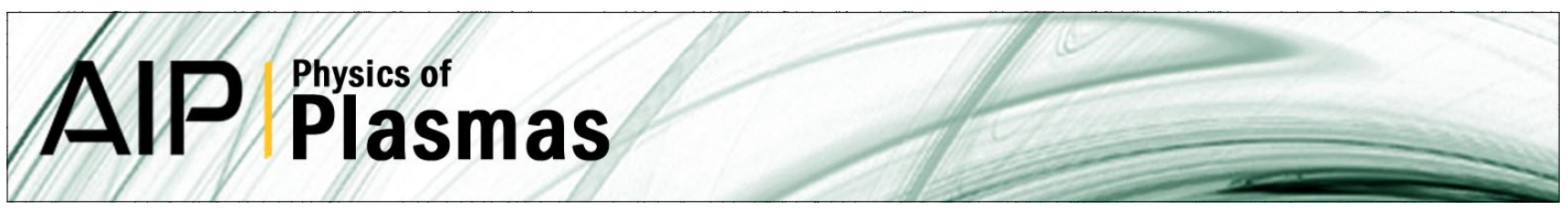

\title{
Plasma kinetics in dusty plasmas
}

Paolo Ricci, Giovanni Lapenta, U. de Angelis, and V. N. Tsytovich

Citation: Phys. Plasmas 8, 769 (2001); doi: 10.1063/1.1344197

View online: http://dx.doi.org/10.1063/1.1344197

View Table of Contents: http://pop.aip.org/resource/1/PHPAEN/v8/i3

Published by the American Institute of Physics.

\section{Related Articles}

Interaction of dust-ion acoustic solitary waves in nonplanar geometry with electrons featuring Tsallis distribution Phys. Plasmas 19, 112302 (2012)

Investigation of the sheath formation in a dusty plasma containing energetic electrons and nano-size dust grains Phys. Plasmas 19, 103505 (2012)

Effect of wake potential on Coulomb crystallization in the presence of magnetic field

Phys. Plasmas 19, 103707 (2012)

On the heterogeneous character of the heartbeat instability in complex (dusty) plasmas

Phys. Plasmas 19, 103701 (2012)

Microparticles deep in the plasma sheath: Coulomb "explosion"

Phys. Plasmas 19, 093709 (2012)

\section{Additional information on Phys. Plasmas}

Journal Homepage: http://pop.aip.org/

Journal Information: http://pop.aip.org/about/about_the_journal

Top downloads: http://pop.aip.org/features/most_downloaded

Information for Authors: http://pop.aip.org/authors

\section{ADVERTISEMENT}

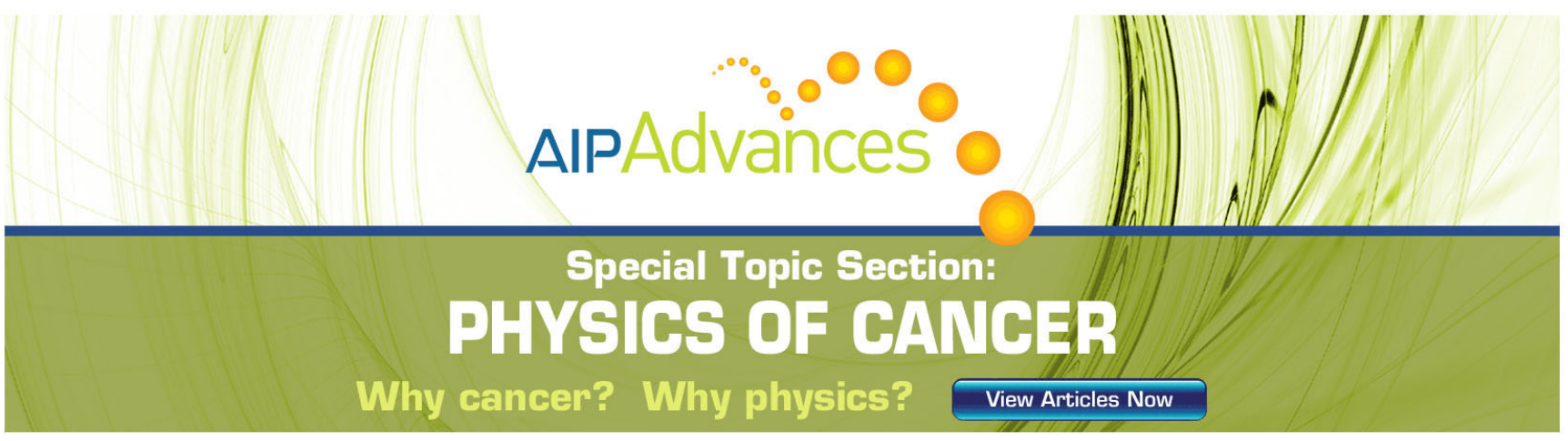




\title{
Plasma kinetics in dusty plasmas
}

\author{
Paolo Ricci and Giovanni Lapenta ${ }^{a)}$ \\ Los Alamos National Laboratory, Los Alamos, New Mexico 87545 \\ $\mathrm{U}$. de Angelis \\ Department of Physical Sciences, University of Napoli “Federico II," Italy \\ V. N. Tsytovich \\ General Physics Institute, Russian Academy of Sciences, Moscow, Russia
}

(Received 10 August 2000; accepted 10 October 2000)

\begin{abstract}
The time evolution of the plasma distribution functions (electrons and ions) in dusty plasmas is found solving numerically the equations of the kinetic theory of dusty plasmas [Tsytovich and de Angelis, Phys. Plasmas 6, 1093 (1999); 7, 554 (2000)]. The role and importance of the plasma source and particle diffusion are investigated. The "equilibrium" distributions (asymptotic solutions) are used to calculate the static screening of a dust particle, and the difference with the usual result (Debye screening) of a three-component plasma is shown for various parameter regimes. (C) 2001 American Institute of Physics. [DOI: 10.1063/1.1344197]
\end{abstract}

\section{INTRODUCTION}

Dusty plasmas are often described using the theory of multicomponent plasmas, the dust particles with a fixed charge $e Z_{d}(e<0)$ being a massive plasma component. In this case, the main differences with the results of threecomponent plasmas arise from the large (sometimes infinite) dust to ion mass ratio and from the possible high values of $Z_{d}$ which can introduce nonlinearities and strong coupling.

Recently, a kinetic theory of dusty plasmas has been formulated ${ }^{1-3}$ taking into account another distinctive feature of these systems: the dust particles must absorb plasma particles (electrons and ions) at all times to maintain their charge and therefore some kind of plasma source must be present to replace the absorbed particles: ionization, plasma reservoir, re-emission from the dust surface, etc. (In the following, Refs. 1-3 will be denoted as Paper I, II, and III, respectively.) As a consequence, the plasma is not collisionless: collisions between plasma particles may or may not be present (they are not taken into account in Papers I-III) but the plasma particles-dust collisions are an essential feature of the kinetic model. As shown in Paper I, the parameter regime where the dust-plasma particles collisions dominate with respect to the collisions between plasma particles is $n_{d} Z_{d}^{2} / n_{e} \gg 1$, where $n_{d}, n_{e}$ are the dust and electron particle densities, respectively. This condition is usually valid in current experiments with dusty plasmas. The charging collisions, of course, alter the dust charge, which is in principle different on each dust particle, depending on the local plasma conditions and fluctuations. This problem has been treated in Paper I, introducing the grain charge $q$ as a dust phase-space variable: the dust particles are described by a time-dependent distribution function of momentum, position, and charge (number of dust particles with charge between $q$ and $q+d q)$. At the same time, the continuous absorption of plasma particles on dust causes changes in the plasma distri-

${ }^{\text {a)} E l e c t r o n i c ~ m a i l: ~ l a p e n t a @ l a n l . g o v ~}$ bution functions (particle diffusion) and their evolution also depends on the properties of the plasma source. The dust charge fluctuations and plasma absorption also modify the space charge around dust particles and one might expect deviations from the usual Debye screening. These effects were discussed in Paper II where Maxwellian distributions were used for numerical estimates of particle diffusion and screening.

In the present work the dust particle distribution is still assumed to be Maxwellian, as it is shown to be a consistent solution of the present kinetic model, but the plasma distributions are assumed Maxwellian only at time $t=0$ (initial values) and the evolution of the electron and ion distributions is found from the numerical solutions of the kinetic equations. These are given in Sec. II in a form suitable for numerical evaluation. In Sec. III, the dependence of the evolution on the plasma source is studied: for Maxwellian sources (a reservoir of Maxwellian plasma) with different intensities, including the case of zero source and the case of a source replacing exactly the number of plasma particles which are absorbed; the solutions of the kinetic equations at different times are plotted for various parameter regimes. The dependence of the plasma evolution on the source can clearly be appreciated in Figs. 1-5. In Sec. IV, the importance of particle diffusion is examined: the evolution of the plasma distributions and corresponding "thermal" energies with and without the diffusion term in the kinetic equations are compared for various parameter regimes. The results confirm the finding of Paper II that diffusion is particularly important for ions and are shown in Figs. 6-8. In Sec. V, the results for the static screening are presented in comparison with the usual Debye screening in a three-component plasma of electrons, ions, and dust particles with fixed charge. Depending on the parameter regime, the "effective" screening, as calculated from the kinetic theory, can be quite different from Debye screening, as already pointed out in Paper II. In particular, the differences at small wave number $k$ are very important 
since they are related to differences in the interaction of two dust particles at large distances. The results are shown in Fig. 9.

\section{KINETIC EQUATIONS FOR ISOTROPIC DISTRIBUTIONS}

The kinetic theory of dusty plasmas (see Papers I, II, III) provides the evolution equations for the distribution functions of all components: electrons, ions, and dust particles. The Maxwellian distribution, however, is a particular solution of the kinetic equation for the dust particles and it is, therefore, assumed in the present calculations where only the coupled equations for electrons and ions are considered. Future work will address the evolution of the dust distribution and its effects on the plasma distributions. In the following, isotropic homogeneous distributions are used for plasma particles $[\alpha=\{e, i\}]$ and dust $(d): f_{\alpha}(\mathbf{r}, \mathbf{v}, t)=f_{\alpha}(v, t)$; $f_{d}\left(\mathbf{r}, \mathbf{v}^{\prime}, t\right)=f_{d}\left(v^{\prime}, t\right)$ normalized to the respective particle densities $n_{i}, n_{e}, n_{d}$. The dust distribution integrated over charge (see Paper I)

$$
f_{d}\left(\mathbf{r}, \mathbf{v}^{\prime}, t\right)=\int f_{d}\left(\mathbf{r}, \mathbf{v}^{\prime}, q, t\right) d q
$$

is assumed to be Maxwellian. We remark that the velocity of dust particles is always denoted with primes to distinguish it from the velocity of plasma particles.

The kinetic equations for the plasma particles can be expressed as

$$
\begin{aligned}
\frac{\partial f_{\alpha}(v, t)}{\partial t}= & S_{\alpha}(v, t)-\nu_{d, \alpha}(v) f_{\alpha}(v, t) \\
& +\frac{\partial}{\partial p_{i}}\left(D_{i, j}^{\alpha, d}(\mathbf{v}) \cdot \frac{\partial f_{\alpha}(v, t)}{\partial p_{j}}\right) \\
& +\frac{\partial}{\partial p_{i}}\left(F_{i}^{\alpha, d}(\mathbf{p}) f_{\alpha}(v, t)\right)+\nu_{d, \alpha}^{f l} f_{\alpha}(v, t) .
\end{aligned}
$$

The term $S_{\alpha}$ represents a regular plasma source of particles of species $\alpha, F_{i}^{\alpha, d}(\mathbf{p})=0$, if the distributions are isotropic, and $\nu_{d, \alpha}^{f l}$, which introduces a collective correction to the absorption on dust given by the second term, is usually small and will be neglected here. The collision frequency and the diffusion coefficient are defined as

$$
\begin{aligned}
\nu_{d, \alpha}(v)= & \iint v \sigma_{\alpha}(q, v) f_{d}\left(v^{\prime}, q, t\right) d q d \mathbf{v}^{\prime} \\
= & \int v \sigma_{\alpha}\left(q_{\mathrm{eq}}, v\right) f_{d}\left(v^{\prime}, t\right) d \mathbf{v}^{\prime}=v \sigma_{\alpha}\left(q_{\mathrm{eq}}, v\right) n_{d}, \\
D_{i, j}^{\alpha, d}(\mathbf{v})= & \frac{2}{\pi} \iint e_{\alpha}^{2}\left|q^{\mathrm{eff}}\left(\mathbf{k}, \mathbf{k} \cdot \mathbf{v}^{\prime}\right)\right|^{2} \\
& \times \frac{k_{i} k_{j}}{k^{4}\left|\epsilon\left(\mathbf{k}, \mathbf{k} \cdot \mathbf{v}^{\prime}\right)\right|^{2}} \frac{\nu_{\alpha, d}(v)}{(\mathbf{k} \cdot \mathbf{v})^{2}+\nu_{\alpha, d}^{2}(v)} \\
& \times f_{d}\left(v^{\prime}, t\right) d \mathbf{k} d \mathbf{v}^{\prime},
\end{aligned}
$$

indicating with $e_{\alpha}$ and $\sigma_{\alpha}$ the charge and the absorption cross section on the dust grains of the particles of species $\alpha$.
In Eq. (1) the approximation that the dust distribution is peaked near the equilibrium charge $q_{\text {eq }}$ has been used, where $q_{\text {eq }}=q_{\text {eq }}(t)$ is the solution of the equation for zero net current

$$
\sum_{\alpha} \int e_{\alpha} v \sigma_{\alpha}\left(q_{\mathrm{eq}}, v\right) f_{\alpha}(v, t) d \mathbf{v}=0 .
$$

In the present work, the orbital motion limited (OML) cross-sections ${ }^{4}$ are used

$$
\begin{aligned}
& \sigma_{i}\left(v, q_{\mathrm{eq}}\right)=\pi a^{2}\left(1+\frac{2 q_{\mathrm{eq}} e}{a m_{i} v^{2}}\right), \\
& \sigma_{e}\left(v, q_{\mathrm{eq}}\right)=\left\{\begin{array}{l}
\pi a^{2}\left(1-\frac{2 q_{\mathrm{eq}} e}{a m_{e} v^{2}}\right) \quad \text { if } v \geqslant \sqrt{\frac{2 q_{\mathrm{eq}} e}{a m_{e}}}, \\
0 \quad \text { if } v<\sqrt{\frac{2 q_{\mathrm{eq}} e}{a m_{e}}},
\end{array}\right.
\end{aligned}
$$

where $m_{\alpha}$ is the mass of particles $\alpha$ and $a$ is the grain radius.

The dielectric function and the effective charge in the diffusion coefficient can be written in the form (see Paper II)

$$
\begin{aligned}
\epsilon(\mathbf{k}, \omega)=1 & +\sum_{\alpha}\left(\epsilon_{\alpha}(\mathbf{k}, \omega)-1\right)+\epsilon_{d}(\mathbf{k}, \omega)-1 \\
q^{\mathrm{eff}}(\mathbf{k}, \omega)= & q_{\mathrm{eq}}+\widetilde{q}(\mathbf{k}, \omega)+\frac{1}{\nu_{\mathrm{ch}}}\left[\widetilde{S}\left(\mathbf{k}, \omega, q_{\mathrm{eq}}, q_{\mathrm{eq}}\right) n_{d}\right. \\
& \left.\times(1+\widetilde{\beta}(\mathbf{k}, \omega))\left(1-\frac{\widetilde{S^{\prime}}\left(\mathbf{k}, \omega, q_{\mathrm{eq}}\right) n_{d}}{\nu_{\mathrm{ch}}}\right)^{-1}\right],
\end{aligned}
$$

with

$$
\begin{aligned}
\epsilon_{d}(\mathbf{k}, \omega)= & 1+\frac{q_{\mathrm{eff}}(\mathbf{k}, \omega)}{q_{\mathrm{eq}}}\left(\boldsymbol{\epsilon}_{d}^{\mathrm{eq}}(\mathbf{k}, \omega)-1\right) \\
& +\frac{4 \pi i S\left(\mathbf{k}, \omega, q_{\mathrm{eq}}\right) n_{d}}{k \nu_{\mathrm{ch}}\left[1-\left(\widetilde{S^{\prime}}\left(\mathbf{k}, \omega, q_{\mathrm{eq}}\right) n_{d} / \nu_{\mathrm{ch}}\right)\right]} \\
& \times(1+\widetilde{\beta}(\mathbf{k}, \omega)),
\end{aligned}
$$

where the charging collision frequency is given by

$$
\nu_{\mathrm{ch}}(t)=-\frac{\partial}{\partial q_{\mathrm{eq}}}\left(\sum_{\alpha} \int e_{\alpha} v \sigma_{\alpha}\left(q_{\mathrm{eq}}, v\right) f_{\alpha}(v, t) d \mathbf{v}\right) .
$$

The plasma responses $S\left(\mathbf{k}, \omega, q_{\mathrm{eq}}\right), \quad \widetilde{S}\left(\mathbf{k}, \omega, q_{\mathrm{eq}}, q_{\mathrm{eq}}\right)$, $\widetilde{S^{\prime}}\left(\mathbf{k}, \omega, q_{\mathrm{eq}}\right), \widetilde{q}(\mathbf{k}, \omega), \epsilon_{\alpha}(\mathbf{k}, \omega), \widetilde{\beta}(\mathbf{k}, \omega)$, and $\epsilon_{d}^{\mathrm{eq}}(\mathbf{k}, \omega)$ are defined in Papers I and II; below an expression useful for numerical computations is calculated in the hypothesis of isotropic distributions.

These responses all have to be evaluated in the kinetic equations for $\omega=\mathbf{k} \cdot \mathbf{v}^{\prime}$ [see Eq. (2)] and, with the exception of $\epsilon_{d}^{\mathrm{eq}}(\mathbf{k}, \omega)$, everywhere $\mathbf{v}^{\prime}$ will appear in factors as $1 /[\mathbf{k}$ $\left.\cdot\left(\mathbf{v}^{\prime}-\mathbf{v}\right)\right]$.

The dust velocity is usually much less than the electron or ion velocities $\left(\mathbf{v}^{\prime} \ll \mathbf{v}\right)$ and consequently, with the exception of $\epsilon_{d}^{\mathrm{eq}}(\mathbf{k}, \omega)$, all the plasma responses can be evaluated for $\omega=0$. Performing the angular integrations, one obtains 


$$
\begin{aligned}
& S\left(\mathbf{k}, 0, q_{\mathrm{eq}}\right)=\frac{4 \pi i}{k} \sum_{\alpha} \frac{e_{\alpha}^{2}}{m_{\alpha}} \int\left[1-\frac{\nu_{d, \alpha}(v)}{k v} \arctan \left(\frac{k v}{\nu_{d, \alpha}(v)}\right)\right] \\
& \times \sigma_{\alpha}\left(q_{\mathrm{eq}}, v\right) v^{2} \frac{\partial f_{\alpha}(v, t)}{\partial v} d v \\
& \widetilde{S}\left(\mathbf{k}, 0, q_{\mathrm{eq}}, q_{\mathrm{eq}}\right)=-\frac{4 \pi}{k} \sum_{\alpha} \int \arctan \left(\frac{k v}{\nu_{d, \alpha}(v)}\right) \\
& \times \sigma_{\alpha}^{2}\left(q_{\mathrm{eq}}, v\right) e_{\alpha} v^{3} f_{\alpha}(v, t) d v, \\
& \widetilde{S}^{\prime}\left(\mathbf{k}, 0, q_{\mathrm{eq}}\right)=-\frac{4 \pi}{k} \sum_{\alpha} \int \arctan \left(\frac{k v}{\nu_{d, \alpha}(v)}\right) \\
& \times \frac{\partial \sigma_{\alpha}\left(q_{\mathrm{eq}}, v\right)}{\partial q_{\mathrm{eq}}} \sigma_{\alpha}\left(q_{\mathrm{eq}}, v\right) e_{\alpha} v^{3} f_{\alpha}(v, t) d v, \\
& \tilde{q}(\mathbf{k}, 0)=-\frac{4 \pi}{k} \sum_{\alpha} \int \arctan \left(\frac{k v}{\nu_{d, \alpha}(v)}\right) \sigma_{\alpha}\left(q_{\mathrm{eq}}, v\right) \\
& \times e_{\alpha} v^{2} f_{\alpha}(v, t) d v, \\
& \epsilon_{\alpha}(\mathbf{k}, 0)=1-\frac{8 \pi^{2} e_{\alpha}^{2}}{k^{2} m_{\alpha}} \int\left(2-\frac{2 \nu_{d, \alpha}(v)}{k v} \arctan \frac{k v}{\nu_{d, \alpha}(v)}\right) \\
& \times \frac{\partial f_{\alpha}(v, t)}{\partial v} v d v \\
& \widetilde{\beta}(\mathbf{k}, 0)=-\frac{4 \pi}{k} \sum_{\alpha} \int \arctan \left(\frac{k v}{\nu_{d, \alpha}(v)}\right) \\
& \times \frac{\partial \sigma_{\alpha}\left(q_{\mathrm{eq}}, v\right)}{\partial q_{\mathrm{eq}}} e_{\alpha} v^{2} f_{\alpha}(v, t) d v .
\end{aligned}
$$

These responses, calculated here for general isotropic distributions, reduce to the expressions given in Paper II for the particular case of Maxwellian distributions. Notice that there are misprints in Paper II which have been pointed out in Paper III. Finally, $\epsilon_{d}^{\mathrm{eq}}(\mathbf{k}, \omega)$ for a Maxwellian dust distribution reduces to the usual expression as given in Paper II.

Also, the diffusion term present in the kinetic equation can be simplified for the case of isotropic distributions. Performing all angular integrations and defining the quantity

$$
\begin{aligned}
I_{1}(k, t) & =\int \frac{f_{d}\left(v^{\prime}, t\right)}{\left|\epsilon\left(k, \mathbf{k} \cdot \mathbf{v}^{\prime}\right)\right|^{2}} d \mathbf{v}^{\prime} \\
& =2 \pi \iint \frac{f_{d}\left(v^{\prime}, t\right)}{\left|\epsilon\left(k, k v^{\prime} \mu\right)\right|^{2}}\left(v^{\prime}\right)^{2} d v^{\prime} d \mu,
\end{aligned}
$$

the diffusion term can be written in the form

$$
\begin{aligned}
\frac{\partial}{\partial p_{i}}\left(D_{i, j}^{\alpha, d}(\mathbf{v}) \cdot \frac{\partial f_{\alpha}(v, t)}{\partial p_{j}}\right) \\
=\frac{8 e_{\alpha}^{2}}{m_{\alpha}^{2} v^{2}} \frac{\partial}{\partial v}\left\{\left[\int\left(\frac{1}{v^{2} k^{2}}-\frac{\nu_{\alpha, d}(v)}{v^{3} k^{3}} \arctan \frac{k v}{\nu_{\alpha, d}(v)}\right)\right.\right. \\
\left.\left.\quad \times\left|q^{\mathrm{eff}}(\mathbf{k}, 0)\right|^{2} I_{1}(k, t) d k\right] v^{2} \nu_{\alpha, d}(v) \frac{\partial f_{\alpha}(v, t)}{\partial v}\right\}
\end{aligned}
$$

To perform the numerical calculations, the kinetic equations are written in a dimensionless form and the values of the dimensionless distribution functions $g_{\alpha}(y, \tilde{t})$ are furnished as the result of the numerical integration. These dimensionless distribution functions $g_{\alpha}(y, \tilde{t})$ are defined as

$$
g_{\alpha}(y, \tilde{t})=\frac{(2 \pi)^{3 / 2} v_{t h, \alpha}^{3}}{n_{\alpha}} f_{\alpha}(v, t)
$$

and they depend on the dimensionless velocity $y$ and on the dimensionless time $\tilde{t}$

$$
y=\frac{v}{\sqrt{2} v_{t h, \alpha}}, \quad \tilde{t}=t \omega_{p e},
$$

where $v_{t h, \alpha}$ is the initial thermal velocity of particles of species $\alpha$ and $\omega_{p e}$ is the initial electron plasma frequency.

In the same manner, the dimensionless sources of the plasma particles, $S_{\alpha}^{a d}$, are defined

$$
S_{\alpha}^{a d}(y, \tilde{t})=\frac{(2 \pi)^{3 / 2} v_{t h, \alpha}^{3}}{\omega_{p e} n_{\alpha}} S_{\alpha}(v, t)
$$

The plasma kinetics depends completely on six dimensionless parameters and thanks to these parameters it is possible to write the kinetic equations in a dimensionless form. The following dimensionless parameters are used:

$$
z=\frac{Z_{d} e^{2}}{a T_{e}}, \quad P=\frac{n_{d} Z_{d}}{n_{e}}, \quad \tau=\frac{T_{i}}{T_{e}}, \quad \tau_{d}=\frac{T_{d}(1+P)}{T_{i} Z_{d} P},
$$

where $T_{\alpha},[\alpha=\{e, i\}]$, are the "thermal" energies of the plasma particles, defined as

$$
T_{\alpha}(t)=\frac{m_{\alpha} \int v^{2} f_{\alpha}(v, t) d \mathbf{v}}{3 n_{\alpha}},
$$

with $T_{\alpha}(0)=m_{\alpha} v_{t h, \alpha}^{2}$. In the same manner, $T_{d}=m_{d} v_{t h, d}^{2}$ is the thermal energy of the dust grains. The other two dimensionless parameters used are the ion Debye length to the dust grain radius ratio, $\lambda_{i} / a$, and the ion to the electron thermal velocity ratio, $v_{t h, i} / v_{t h, e}$.

We observe that the last dimensionless parameter, $v_{t h, i} / v_{t h, e}$, can be chosen in order to make the net current on the dust grains zero at the starting time.

In fact, Eq. (3), for $t=0$, using the dimensionless parameters, becomes 

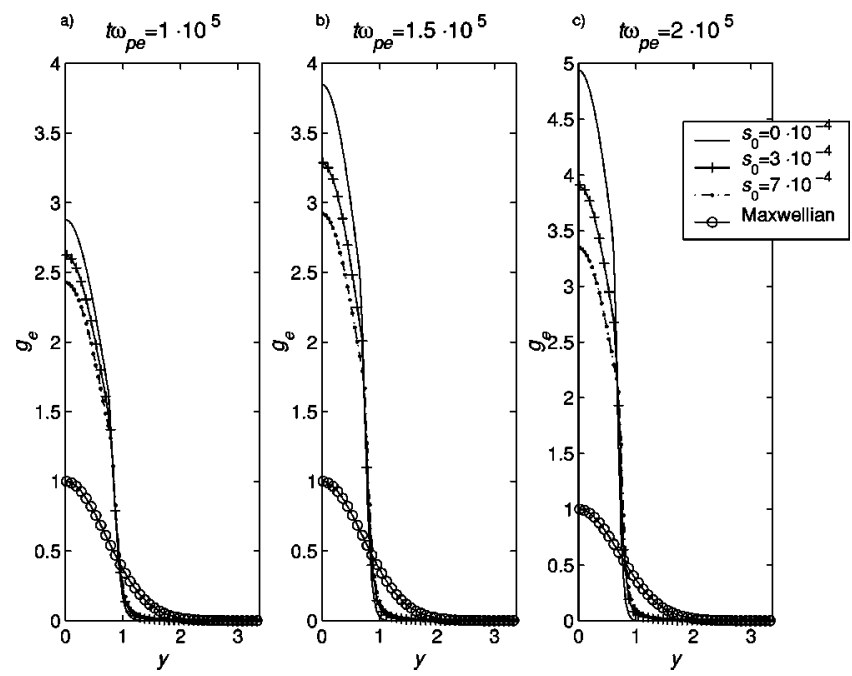

FIG. 1. Normalized electron distribution function vs normalized velocity at different times for different values of the source intensity.

$$
\begin{aligned}
& n_{i} v_{t h, i} \int_{0}^{\infty}\left(1+\frac{z}{\tau y^{2}}\right) g_{i}(y, 0) y^{3} d y \\
& \quad=n_{e} v_{t h, e} \int_{\sqrt{z}}^{\infty}\left(1-\frac{z}{y^{2}}\right) g_{e}(y, 0) y^{3} d y,
\end{aligned}
$$

because the OML cross sections, with the notation $q_{\text {eq }}$ $=e Z_{d}$, can be expressed as

$$
\begin{aligned}
& \sigma_{i}\left(v, q_{\mathrm{eq}}\right)=\pi a^{2}\left(1+\frac{z}{\tau y^{2}}\right), \\
& \sigma_{e}\left(v, q_{\mathrm{eq}}\right)=\left\{\begin{array}{l}
\pi a^{2}\left(1-\frac{z}{y^{2}}\right) \quad \text { if } y \geqslant \sqrt{z}, \\
0 \quad \text { if } y<\sqrt{z} .
\end{array}\right.
\end{aligned}
$$

Using the neutrality condition $n_{i} / n_{e}=1+P$, it is then possible to calculate the dimensionless parameter

$$
\frac{v_{t h, i}}{v_{t h, e}}=\frac{1}{1+P} \frac{\int_{\sqrt{z}}^{\infty}\left(1-\frac{z}{y^{2}}\right) g_{e}(y, 0) y^{3} d y}{\int_{0}^{\infty}\left(1+\frac{z}{\tau y^{2}}\right) g_{i}(y, 0) y^{3} d y},
$$

so that the net current on the dust grains is zero at the starting time.

The two coupled evolution equations for the electrons and for the ions are integrated numerically with the initial conditions that $g_{\alpha}(y, 0)$ are Maxwellian distributions and for several choices of the plasma sources. Simultaneously, the equation for the dust charge is solved self-consistently, taking into account the number of electrons and the number of ions absorbed by the dust grains.

The numerical integration of the kinetic equations has been performed approximating the differential operators by finite differences and then solving the resulting difference equations in an evolutionary manner, starting from $t=0$. Two numerical schemes have been implemented. In the first,
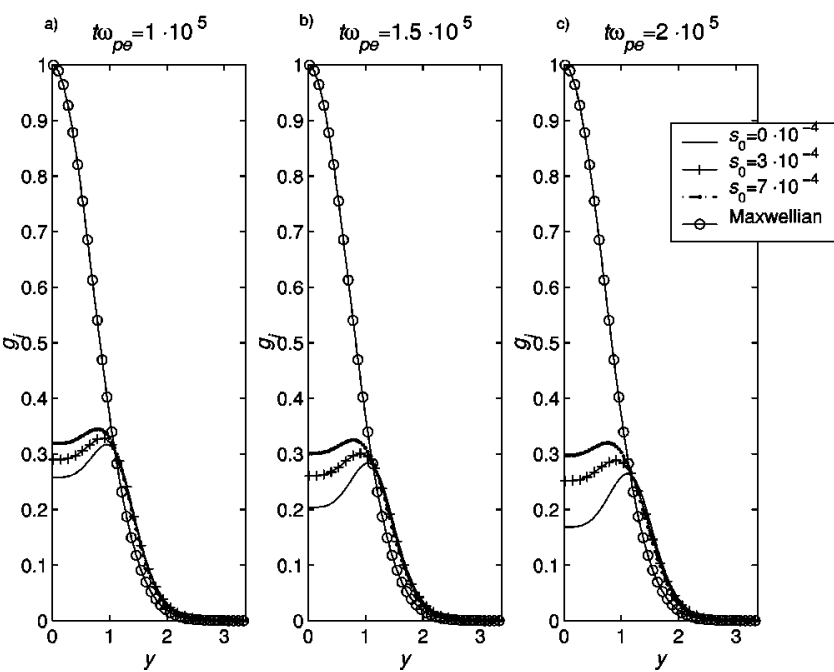

FIG. 2. Normalized ion distribution function vs normalized velocity at different times for different values of the source intensity.

the time derivative has been approximated by a forward difference, in the second, by a backward difference. The results of the two methods have been compared to evaluate the numerical error of the solutions.

\section{IMPORTANCE OF THE PLASMA SOURCE}

To understand how important the source of plasma particles is in the time evolution of the plasma distributions, Maxwellian sources are chosen with different intensities.

The sources have the form

$$
S_{\alpha}(v, t)=s_{0}(t) n_{e} \omega_{p e} \frac{\exp \left[-1 / 2\left(v / v_{t h, \alpha}\right)^{2}\right]}{v_{t h, \alpha}^{3} \sqrt{(2 \pi)^{3}}},
$$

so that the same number of electrons and ions is introduced in the plasma (to preserve the neutrality). The dimensionless sources can be expressed as
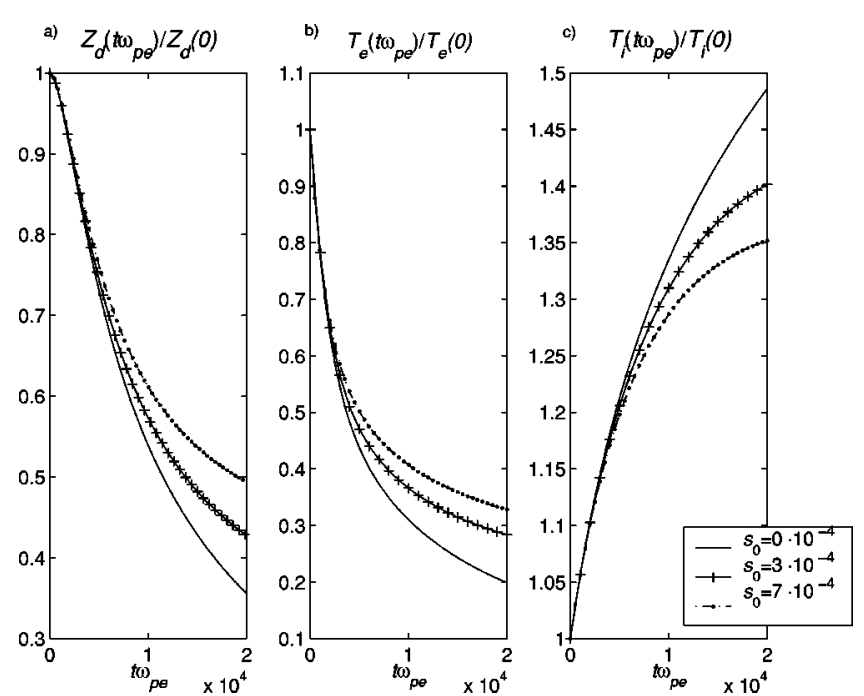

FIG. 3. Evolution of the dust charge (a), electron temperature (b), and ion temperature (c), all normalized to the initial value, as calculated from the distribution functions of Figs. 1 and 2. 
TABLE I. Starting parameters for the simulations presented in Figs. 1, 2, 3, 4(a), and 5(a).

\begin{tabular}{cccccc}
\hline \hline$z$ & $P$ & $\tau$ & $\tau_{d}$ & $\lambda_{i} / a$ & $v_{t h, i} / v_{t h, e}$ \\
\hline 1 & 1.5 & 0.01 & 1 & 50 & $1.45 \cdot 10^{-3}$ \\
\hline \hline
\end{tabular}

$$
S_{e}^{a d}(y, \tilde{t})=s_{0}(\tilde{t}) e^{-y^{2}}, \quad S_{i}^{a d}(y, \tilde{t})=s_{0}(\tilde{t}) \frac{e^{-y^{2}}}{1+P},
$$

for four cases $s_{0}=0$ (no source present); $s_{0}=3 \cdot 10^{-4} ; s_{0}$ $=7 \cdot 10^{-4} ; s_{0}(\tilde{t})=s(\tilde{t})$, the last one chosen so that the number of electrons absorbed is reintroduced in the system.

The kinetic equations are solved for different values of the initial $(t=0)$ parameters: $z, P, \tau, \tau_{d}, \lambda_{i} / a, v_{t h i} / v_{t h e}$. Figures 1 and 2 show snapshots at different times of the distribution functions for the first three values of the source. Figure 3 shows the evolution of the plasma thermal energies, $T_{\alpha}$, normalized to the initial values, and of the dust charge, $Z_{d}$, obtained computing the fluxes of ions and electrons collected by the dust particle. The starting parameters used in Figs. 1-3 are given in Table I.

It is clear that the evolution of the distributions depends strongly on the sources, and the differences grow with time. Even if the electron and ion initial distributions are Maxwellian and the plasma parameters are chosen so that the net current on the dust grains is zero at $t=0$, the distributions of ions and electrons do not maintain their Maxwellian shape. This is still more remarkable because the sources are also Maxwellian. Notice from Fig. 3 that the electron temperature decreases with time because only the energetic electrons are absorbed on the dust grains. This is more relevant in the case without source because the energetic electrons captured are not replaced by a source. The opposite happens for the ion temperature. From the evolution of the dust charge in Fig. 3 it can be seen that the initial dust charge is an equilibrium
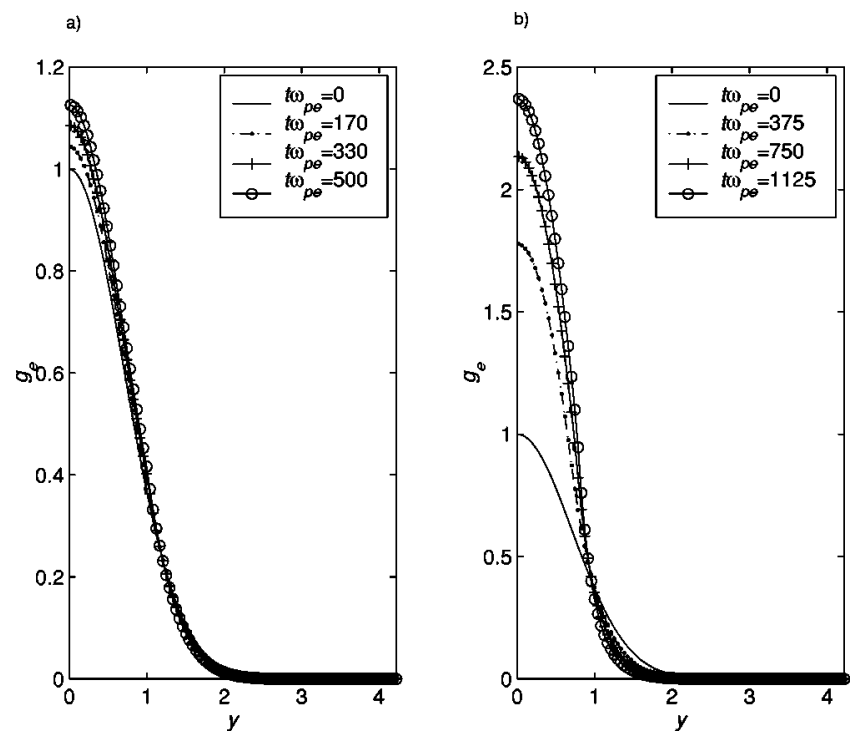

FIG. 4. Normalized electron distribution function vs normalized velocity at different times for the variable source $s_{0}\left(t \omega_{p e}\right)=s\left(t \omega_{p e}\right)$. Starting parameters of Table I (a), starting parameters of Table II (b).
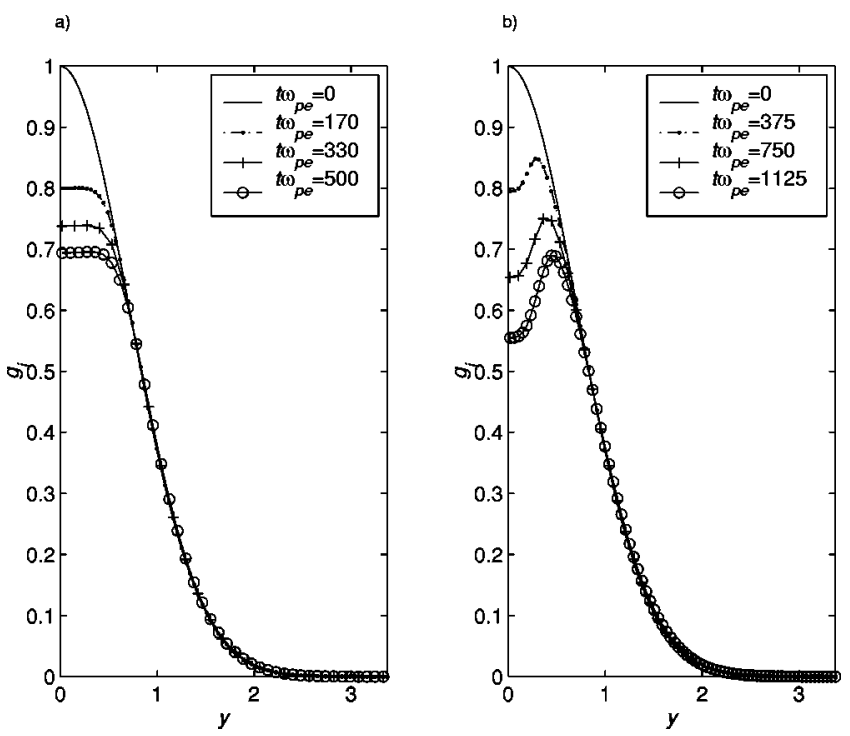

FIG. 5. Normalized ion distribution function vs normalized velocity at different times for the variable source $s_{0}\left(t \omega_{p e}\right)=s\left(t \omega_{p e}\right)$. Starting parameters of Table I (a), starting parameters of Table II (b).

charge only for the initial plasma distribution functions but, as the shapes of the plasma particle distributions change with time, the dust equilibrium charge (which is a function of the electron and ion distributions) changes.

For the case of a variable source, $s_{0}(\tilde{t})=s(\tilde{t})$, which replaces the electrons absorbed by the dust grains so that the electron density is a constant, the evolution of the distribution functions is followed until a stationary solution is reached. Note that a stationary solution for the electrons cannot be reached at low energy because, while the source introduces particles, in this energy region electrons are not absorbed and, as shown in the next section, the diffusion term is negligible for electrons: however, the shape of the ion distribution reaches its stationary solution and so does the electron distribution at high energy. Two cases are shown in Figs. 4 and 5 for different starting parameters and a variable source. The first case [Figs. 4(a),5(a)] has the same parameters of the previous cases (listed in Table I), while in the second case [Figs. 4(b),5(b)] the initial parameters are given in Table II.

The stationary solutions are different in the two cases.

These results show that it is not possible to assume that the electron and ion distributions in a dusty plasma are Maxwellian. Even if the initial distributions and the sources are Maxwellian, the Maxwellian shape of the distribution functions is not maintained in the evolution.

TABLE II. Starting parameters for the simulations presented in Figs. 4(b) and 5(b)

\begin{tabular}{cccccc}
\hline \hline$z$ & $P$ & $\tau$ & $\tau_{d}$ & $\lambda_{i} / a$ & $v_{t h, i} / v_{t h, e}$ \\
\hline $1 \cdot 10^{-2}$ & $1.5 \cdot 10^{-2}$ & 0.1 & $1 \cdot 10^{-2}$ & 50 & $1.33 \cdot 10^{-2}$ \\
\hline \hline
\end{tabular}




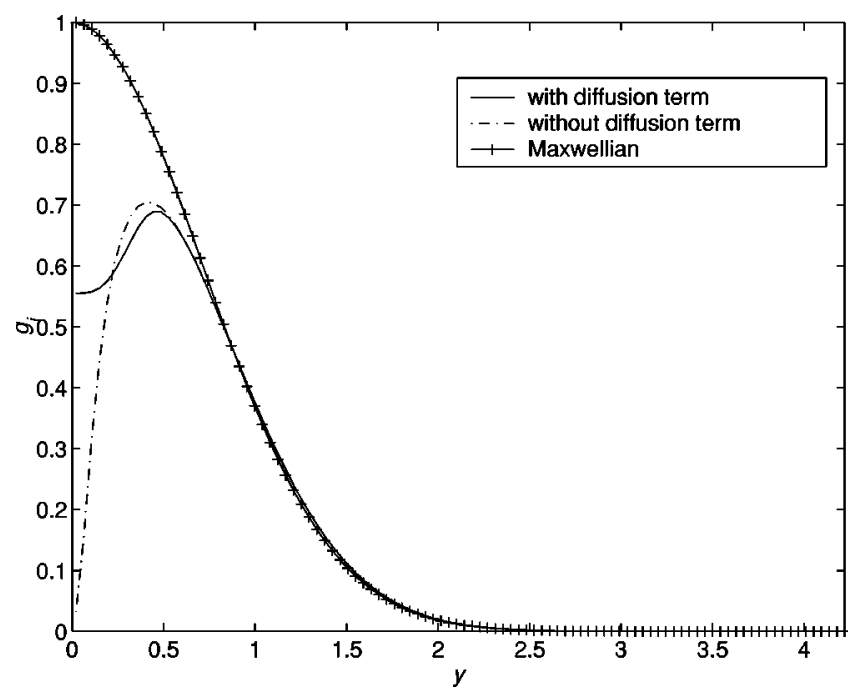

FIG. 6. Normalized ion distribution function vs normalized velocity at $t \omega_{p e}=1125$ from the solutions with and without the diffusion term. The initial Maxwellian is shown for comparison. The starting parameters are given in Table II and the source is variable: $s_{0}\left(t \omega_{p e}\right)=s\left(t \omega_{p e}\right)$.

\section{IMPORTANCE OF THE DIFFUSION TERM}

It was argued in Paper II, where Maxwellian distributions were used for the estimates, that particle diffusion resulting from the absorption processes should be of particular importance for ions but not for electrons. This has been investigated comparing the evolution of the distributions from the numerical solutions with and without the diffusion term in the kinetic equations. The results confirm the findings of Paper II and are shown in Figs. 6-8 for ions.

In Fig. 6 the final ion distributions obtained with and without the diffusion term are compared. For the initial parameters of Table II the time evolution has been followed with a variable source up to $\tilde{t}=1125$. The shapes of the two distributions are clearly different, and the ion distribution function seems to be more affected by the diffusion term at low energy. The diffusion term, in fact, is greater at low energy and its main effect on the shape of the distribution function is to prevent a great depletion of ions at low energy.

As shown in Paper II, another effect of the diffusion term is a growth of the ion temperature. The temperature growth is due both to the diffusion term and to the absorption by dust grains. The temperature growth, described by the diffusion term, is caused by the inelasticity of the dustplasma collision in presence of charging process (see Papers I, II).

To understand the importance of the diffusion term, calculations have been made for several values of the plasma parameter $P$, which plays an important role in the plasma kinetics. The results are shown in Fig. 7 and Fig. 8 for the case of the variable source and starting parameters of Table I (except the value of $P$, which is now varied). In Fig. 7 the ion temperatures are shown as calculated from the solutions for the ion distributions with and without the diffusion term.

With all the other parameters fixed, the evolution of the plasma particles with and without the diffusion term, for $P$ $=0.1[$ Fig. 7(a)], $P=0.5$ [Fig. 7(b)] and $P=1.5$ [Fig. 7(c)],

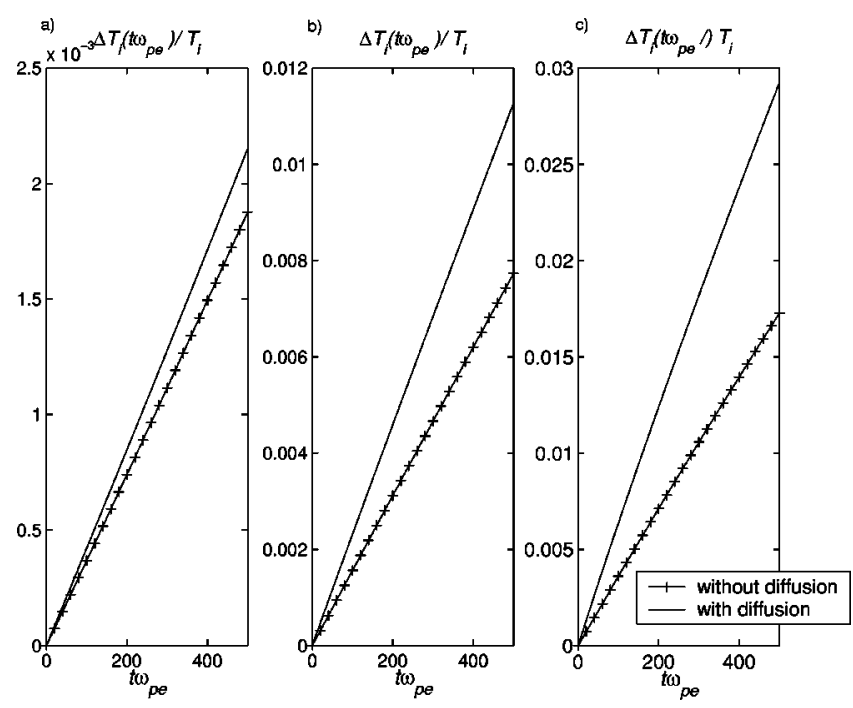

FIG. 7. Growth of the ion temperature (normalized to the initial value) calculated from the distribution functions with and without the diffusion term for three values of the parameter $P$ : $P=0.1$ (a), $P=0.5$ (b), $P=1.5$ (c). The other starting parameters are given in Table I.

has been followed up to $\tilde{t}=500$. In Fig. 8 the total growth of the ions temperature, $\Delta T_{i}$, is compared with the growth caused by the diffusion term, $\Delta T_{i}^{\text {inel }}$ (see Paper II). It is clear that the importance of the diffusion term is nearly constant in time and it grows with $P$ : the last result could also be obtained from the findings of Paper II. For $P$ of the order of 1 , as found in Paper II, the diffusion term is important and the growth of the ion temperature due to the diffusion term is nearly the same as the growth caused by the charging process.

The effect of the diffusion term on the electron distribution function has also been investigated and found to be small. The growth of the electron temperature $\Delta T_{e}^{\text {inel }}$ is very small compared to the electron cooling caused by the charging process $\Delta T_{e}: \Delta T_{e}^{\text {inel }}$ is only of the order of $0.01 \%$ of

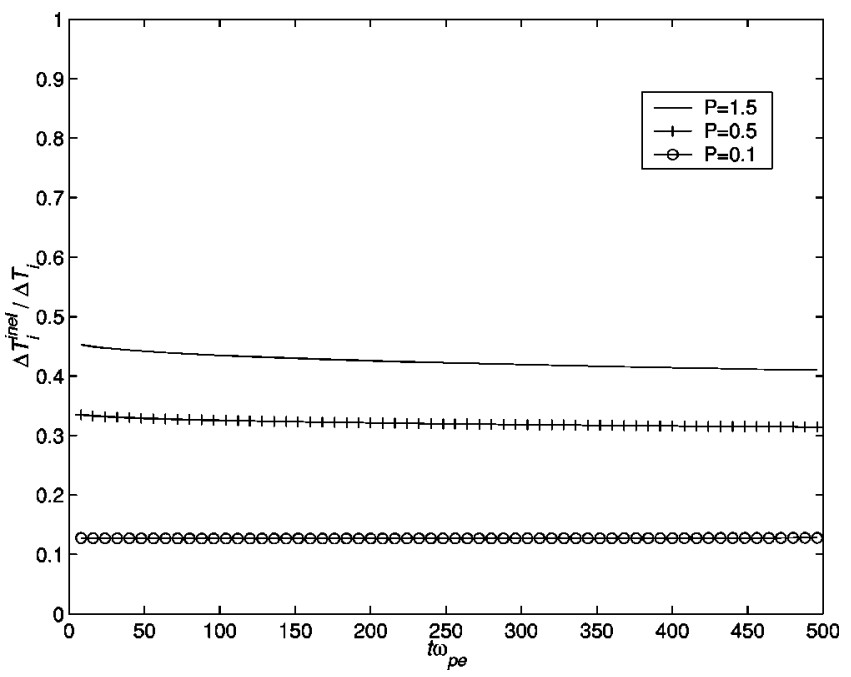

FIG. 8. Dependence on the parameter $P$ of the growth of the ion temperature $\Delta T_{i}^{\text {inel }}$ due to diffusion, normalized to the total growth $\Delta T_{i}$ due to both diffusion and absorption on dust. The starting parameters are the same as Fig. 7. 
$\Delta T_{e}$. Also, in the electron case the importance of the diffusion term seems to grow with $P$, but no meaningful results can be presented to show the importance of the diffusion term for different starting parameters, as the effect of the diffusion term is comparable with the numerical error.

In conclusion, while the diffusion term does not have an important effect on the electron temperature, it cannot be neglected in the evolution of the ion temperature. The iondust inelastic collisions are important and cause a growth of the ion temperature which is comparable with the effect of the charging collisions. This effect grows with $P$ and for $P$ of the order of 1, a typical value in dusty plasmas, the temperature growth due to diffusion is nearly the same as that due to the charging collisions.

\section{STATIC SCREENING}

As shown in Paper III, the interaction between two dust particles at rest in a dusty plasma can be written in terms of an effective potential around a dust particle given by

$$
\varphi(k)=\frac{4 \pi q_{\mathrm{eq}}}{k^{2} \epsilon^{\mathrm{eff}}(k, 0)},
$$

where

$$
\epsilon^{\mathrm{eff}}(k, 0)=q_{\mathrm{eq}} \frac{\epsilon(k, 0)}{q^{\mathrm{eff}}(k, 0)} .
$$

This expression can be derived from the kinetic theory using test-particle theory and it contains all the effects of space-charge modifications due to plasma absorption on dust and charge fluctuations. If the dusty plasma is treated as a three-component plasma, with dust particles of fixed charge $q_{\text {eq }}$, the effective dielectric function is replaced by the usual form

$$
\epsilon^{p}(k, 0)=1+\frac{k_{\mathrm{tot}}^{2}}{k^{2}},
$$

where $k_{\text {tot }}$ is the inverse of the total Debye length of the plasma, including the dust Debye length

$$
k_{\mathrm{tot}}^{2}=\lambda_{i}^{-2}+\lambda_{e}^{-2}+\lambda_{d}^{-2} \text {. }
$$

As is well known, the Fourier transform of the latter gives the Debye-Huckel screened potential

$$
\varphi^{p}(r)=\frac{q_{\mathrm{eq}}}{r} e^{-k_{\mathrm{tot}}},
$$

which has often been used to describe the interactions in dusty plasmas. It is interesting, therefore, to understand the differences between the potential for the present kinetic theory and for a multicomponent plasma and the differences between the two corresponding screening factors. In particular, since the Coulomb interactions are screened but the interaction (change of momentum) due to plasma bombardment is not, one should expect a very different behavior at large distances (small $k$ ) where eventually the potential can change sign (attraction). Since the screening factor depends on the plasma distribution functions, it will change with
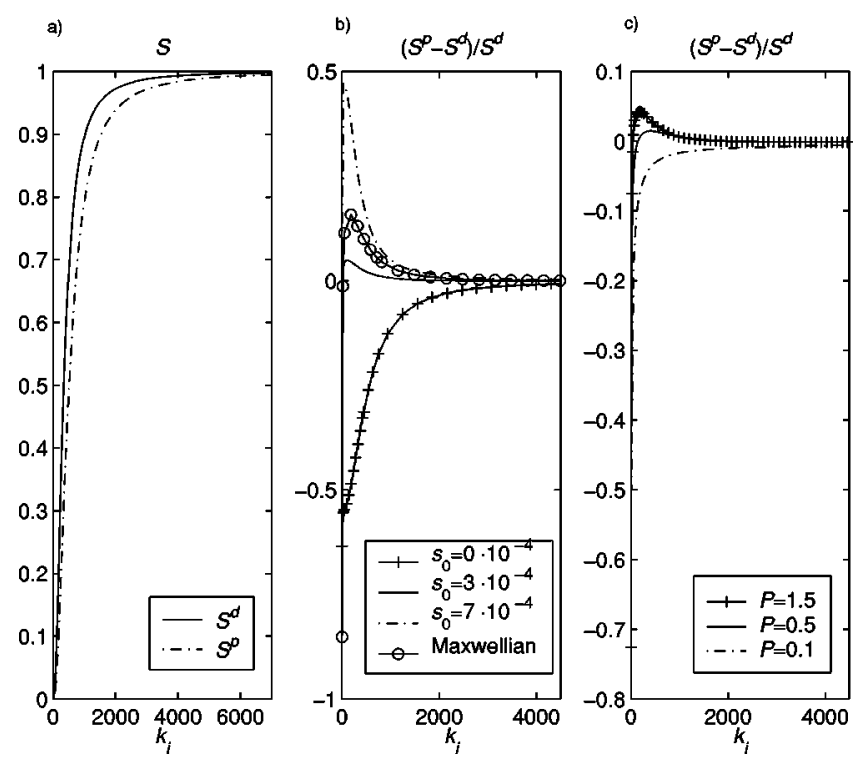

FIG. 9. Static screening of a dust particle from the kinetic theory $\left(S^{d}\right)$ and from the theory of multicomponent plasmas $\left(S^{p}\right)$ vs normalized wave number $k_{i}$. For the parameters of Table I, $s_{0}=7 \cdot 10^{-4}$ and $t \omega_{p e}=2 \cdot 10^{5}$ (a); for different values of the source intensity, starting parameters of Table I and $t \omega_{p e}=2 \cdot 10^{5}(\mathrm{~b})$; for the starting parameters of Table II, variable source $s_{0}\left(t \omega_{p e}\right)=s\left(t \omega_{p e}\right), t \omega_{p e}=500$, and three values of $P(\mathrm{c})$.

time. The following numerical results refer to the "equilibrium" case in the sense that the asymptotic plasma distributions are used to calculate the effective dielectric function. The results are shown in Fig. 9.

Introducing the dimensionless wave number

$$
k_{i}=\frac{\tau}{\pi a^{2} n_{d} z} k,
$$

it is

$\epsilon^{p}\left(k_{i}\right)=1+\left[1+\frac{\tau}{1+P}+\frac{1}{\tau_{d}}\right] \frac{1}{\left(a / \lambda_{D i}\right)^{2}\left[P^{2} / 16(1+P)^{2}\right] k_{i}^{2}}$.

In Fig. 9 the static screening in a dusty plasma

$$
S^{d}\left(k_{i}\right)=\frac{q^{\mathrm{eff}}\left(k_{i}\right)}{q_{\mathrm{eq}} \epsilon\left(k_{i}, 0\right)},
$$

is compared with the static screening in a multicomponent plasma

$$
S^{p}\left(k_{i}\right)=\frac{1}{\epsilon^{p}\left(k_{i}, 0\right)},
$$

for various parameter regimes.

In Fig. 9(a) the screening is calculated from the distributions found for the cases presented in Figs. 1 and 2 (in the case $s_{0}=7 \cdot 10^{-4}$ ). The distributions used are those obtained at the end of the calculation, when $\tilde{t}=2 \cdot 10^{5}$. It is clear that the difference between the screenings is remarkable at small $k$, and decreases when $k$ grows, as predicted. Most important is that the analytic form is different for small $k$ (as shown in Paper III) which means that the simple Debye-Huckel form of the potential is not valid at large distances. 
The screening $S^{p}$ has been evaluated with the final density and temperature of the plasma particles and with the final charge of the dust particles.

In this dusty plasma $S^{p}$ is always smaller than $S^{d}$, but this is not true in all dusty plasmas, as the differences between the two screenings change in an essential manner, for various parameter regimes. This is shown in Fig. 9(b), where the relative differences between the two screenings are plotted for all the sources of Figs. 1 and 2. The distributions used are again those at $\tilde{t}=2 \cdot 10^{5}$. The case of Maxwellian distributions is also shown in Fig. 9(b): the screening $S^{d}$ in this case has been calculated not from the solutions of the kinetic equations but by using Maxwellian distributions for the plasma particles. As shown by a comparison with the other curves, the difference in screening can be enhanced when the consistent solutions of the kinetic equations are used. It is remarkable that $S^{d}$ can be smaller or greater than $S^{p}$ and that the difference between the two screenings can change sign. The differences between the two screenings are important for small $k$ and when $k$ grows the differences reduce. The differences between the two screenings can be great, and a positive peak of $50 \%$ is visible when $s_{0}=0$. For very low values of $k$, the differences between the two screenings are always great and negative ( $S^{d}$ is very small and a difference is amplified when relative differences are plotted).

The values of $k_{i}$ must be compared with $k_{\max } \simeq 1 / a,{ }^{5}$ that is

$$
k_{i, \max }=\frac{\tau}{z \pi a^{3} n_{d}}=4\left(\frac{\lambda_{i}}{a}\right)^{2} \frac{1+P}{P},
$$

and, with the starting parameters of the simulations, it is $k_{i, \max } \approx 1.7 \cdot 10^{4}$. In Fig. 9(c) the parameters of Table II are used with a variable source and the evolutions of the distributions have been followed up to $\tilde{t}=500$. The different behavior of the differences between the two screenings, for the various values of $P$, is apparent. The differences can be positive or negative.

The differences depend strongly on the dusty plasma parameters and different qualitative effects can be seen for various parameters. The differences at small $k$ imply that around a dust charge the potential does not have the usual Debye screened form for large $r$. The numerical study of this potential will be the subject of a future work.

\section{CONCLUSIONS}

In the parameter regime where dust-plasma particles collisions dominate with respect to collisions between plasma particles and the recent kinetic theory of dusty plasmas (Paper I,II,III) is valid, the evolution equations for the distributions of electrons and ions have been solved numerically with the assumptions of isotropic and homogeneous functions and of a Maxwellian dust distribution at all times.

The importance of the source and diffusion of plasma particles have been investigated with the conclusion that, even for Maxwellian sources, the plasma equilibrium distributions are not Maxwellian and the ion and electron initial temperatures change due to absorption on dust and diffusion (see Figs. 1-5). The effect of diffusion has been shown to be important for ions (see Figs. 6-8) but not for electrons, as anticipated in Paper II.

Finally, the static effective screening of a dust particle, as calculated from the results of the kinetic theory including dust charge fluctuations and the modification of the space charge due to plasma absorption on dust, has been shown to be quite different from the usual Debye screening of multicomponent plasmas at small wave numbers (see Fig. 9). This implies that the interaction of two dust particles in a dusty plasma can have, at large distances, a very different behavior from the usual screened Coulomb interaction. A detailed analysis of this interaction will be the subject of a future work.

\section{ACKNOWLEDGMENTS}

Work performed under Contract No. HPRN-CT200000140 from the European Community. One of the authors (V.N.T.) would like to thank the Max Planck Institute for Extraterrestrial Physics (Garching, Munich) for the hospitality and acknowledge the support of an A. von Humboldt award.

${ }^{1}$ V. N. Tsytovich and U. de Angelis, Phys. Plasmas 6, 1093 (1999).

${ }^{2}$ V. N. Tsytovich and U. de Angelis, Phys. Plasmas 7, 554 (2000).

${ }^{3} \mathrm{~V}$. N. Tsytovich and U. de Angelis, "Kinetic theory of dusty plasmas. III. Dust-dust collision integrals,"’ Phys. Plasmas (to be published).

${ }^{4}$ J. E. Allen, Phys. Scr. 45, 497 (1992).

${ }^{5}$ S. Benkadda, P. Gabbai, V. N. Tsytovich, and A. Verga, Phys. Rev. E 53, 2717 (1996) 